\title{
ON THE USE OF TUBES OF COLORED GLASS FOR NES- SLERIZING.
}

\section{By A. A. Brenemas.}

The idea of using the ordinary amber-colored window glass of lifferent shades to furnish a series of standard tints for use in the Nessler ammonia test, occurred to me several years ago, and a note upon the subject was read at the Boston meeting of the American Association in 1880. Since then I have found, however, that the suggestion was not essentially new, and the method proposed moreover, that of holding a slip of glass of the desired tint over the mouth of a Nessler test tube filled to the mark with clear water while the tube containing the unknown quantity of ammonia is beld by the side of the test tube for comparison, is open to objection. The fact that light coming to the eye after passing through the length of the comparison tube is modified by reflection at the surface of the colored glass, as also the fact that the colored media in the two tubes have their upper surfaces respectively in different planes and at different distances from the eye, both tend to prevent a satisfactory comparison of the two tints. While these difficulties might be corrected, in part at least, by surrounding each tube with a blackened sheath or case, it has seemed more in accordance with the ordinary method of making the Nessler test and with the principles of cololimetry in general, to introduce the colored medium be low the comparison tube. The light from this colored mediun should be applied in connection with a tube precisely like the comparison tube and filled to the unark with clear water; in no other way can the quality of illumination, which is essential to a fair comparison, be obtained. The suitability of glass as the colored medium is simply a question of securing the proper tints. Its cleanliness, uniformit and fixity of tint render it far superior to any other substance that could be applied to this purpose. It was mentioned in the note re. ferred to that the tints of commercial amber glass corresponded very well with the higher values needed among the Nessler standards, such for example as the tints given by lc.c. of Wanklyn's weak solution of ammonia (1c.c. $=.0000 \mathrm{l} \mathrm{grm} . \mathrm{NH}_{3}$ ), but it was found difficult to obtain glass matching the tints given by smaller quantities of ammonia and for values under 0.5c.c. of the standard solution, no glass of tint sufficiently light could be found. Even "flashed" 
glass which might be supposed to yield lighter tints than "potcolored" glass, is still too dark for the purpose. The manufacture of glass is too costly an operation to permit of experiments being made upon special "batches" of glass made for the purpose, unless some manufacturer could be inspired with a seientific interest in such work, or were given an order large enough to warrant the attempt as a matter of business. Failing in this direction, it oecurred to me that a tube made from glass of the proper tint might answer the purpose, and the fiact that the bottom of such a tube would be blown, suggested the means of varying its thickness, and therefore its tint, to any desired extent. Such a tule was found, however, to be unsuitable for comparison with the color of Nessleriyed liquids, because the yellow light coming through the sides of the tube increased the color given to the light coming through the bottom, and even when filled with clear waters the quality of illumination in the two tubes was such as to forbid comparison. The only suitable con. dition seems to be that in which the light enters the tube through the colored medium placed at or very near the bottom of the tube. The sides of the tube should be of colorless glass, and the tuhe filled with clear water as mentioned above. These conditions secm to be best fulfilled by such tubes as are shown here, for which I am indebted to the cooperation of a skillful glass-blower, Mr. William Baetz. $A$ short length of amber glase tubing is fused to a longer piece of coloress tubing to form a tube like those used in Nesserlizing. The bottom is flat, and the colored portion forms about one-fifth of the entile tube. It is a matter of come difficulty to find glass of proper composition to adhere readily to the amber glass, and still more so to extend the colored part of the cylinder by blowing without altering its dinmeter. Both result have been fairly accomplished however, and slight defects shown in these tubes will be removed, no doubt, after practice in making them. It is possible, then, to start with glass tubing of a given shade and to weaken the tint by blowing out the tube until the desired tint is reached. The tube is then closed and flattened at the desired distance from the junction with the coloress glass. In practice $I$ have found it more simple to have a number of such tules made of various shades below a certain one and to pick out from these such as corresponded exactly with the different values given by the Nessler reagent with known quantitics of ammonia. It has been my custom for years in using Wanklyn's method 
of water analysis, to distill off 10c.c. at a trial, using only 100c.c. of the water to be tested, and all of these tubes have been made of the 10c.c. size, but the principle is perhaps more easily applicable tolarger tulues, because the variations of tints required would be less delicate. For very light shades I have found an amber glass with a barely perceptible olive tint to yield the best results when distended and weakened in color as described. It should be said finally, that the junction of the compound tube causes an unavoidable ringed appearance in the tube when filled with water, but with a well-made bottom to the tube there is always a clear space within the innermost circle sufficiently large to permit of comparison with the tint of the other tube.

\section{ON THE ANALYSIS OF A SAMPLE OF MINERAL WATER FROM IRONDALE, WEST VIRGINIA.}

\section{By A. A. Breneman.}

I have recently had occasion to examine a sample of mineral water which, as shown by the accompanying analysis, is somewhat peculiar in composition. The water was taken from a spring on the property of Mr. F. Nemegyei, at Irondale, Preston Co., West Virginia. This spring issues near the base of a hill in which beds of bituminous coal alternate with beds of fine clay, limonite and limestone. The coal in some of the veins, especially in the higher part of the hill, is very rich in pyrites.

The water has a faintly acid reaction, and in taste is weakly astringent. Its color is perceptibly yellowish in thick layers.

Analysis yields the following results expressed in grains per U. S. gallon :

Calcium Sulphate

Aluminium "

Potassium "

Magnesium "

Manganese "

Ferrous (?) "

Cobaltous "s

Sodium Chloride.

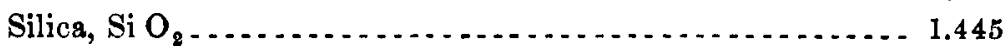

Nitric Acid 